\title{
Junctional epidermolysis bullosa, generalized intermediate
}

INSERM

\section{Source}

INSERM. (1999). Orphanet: an online rare disease and orphan drug data base. Junctional epidermolysis bullosa, generalized intermediate. ORPHA:79402

Generalized non-Herlitz-type junctional epidermolysis bullosa is a form of non-Herlitztype junctional epidermolysis bullosa (JEB- $\mathrm{nH}$, see this term) characterized by generalized skin blistering, atrophic scarring, nail dystrophy or nail absence, and enamel hypoplasia, with extracutaneous involvement. 